\title{
Taking a new TAK on Tat transactivation
}

\author{
Katherine A. Jones ${ }^{1}$
}

The Salk Institute for Biological Studies, La Jolla, California 92037-1099 USA

A common ratelimiting step in the transcription of inducible eukaryotic genes is the release of RN A polymerase II (RN APII) molecules from stalled elongation complexes that accumulate shortly after the initiation of RNA synthesis (Blair et al. 1996; Blau et al. 1996; for review, see Greenblatt et al. 1993; Bentley 1995). In the absence of the inducer protein, transcription at these genes is usually initiated by RN AP complexes that pause at sites 20-60 nucleoti des downstream of the promoter. Promoter-proximal pausing is then counteracted by DNA- or RNA-binding activators that are capable of recruiting or stimulating positive-acting transcription elongation factors. Control at the level of elongation allows for the rapid induction of transcription in response to a specific activator, in part by bypassing the need to assemble stable preinitiation complexes and also by maintaining the promoter in an open conformation that is immediately accessible to regulatory factors.

The induction of the human immunodeficiency virus (HIV-1 and HIV-2) promoters by the essential virus-encoded $T$ at protein has proven to be a very useful model system for studies of the mechanisms that regulate the assembly and release of early el ongation complexes. In this issue of Genes \& Development, three exciting new papers provide the strongest evidence to date that the HIV Tat proteins act to target host cell kinases that enhance the processivity of RN APII at an early elongation step. First, Zhu et al. (this issue) demonstrate that Tat binds with high affinity and specificity to a novel carboxy-terminal domain (CTD) kinase complex, the positive transcriptional el ongation factor $b$ (P-TEFb) known to function at an early elongation step, and that the protein kinase responsible for P-TEFb activity is a previously known cdc2-related kinase called PIT ALRE (Grana et al. 1994; Garriga et al. 1996). Second, Mancebo et al. (this issue) report that potent new chemical inhibitors of Tat isolated from random drug screens appear to target the active site of the PITALRE kinase. This remarkable convergence of evidence on the role of a new CTD kinase marks a major advance in our understanding of the mechanism of Tat activation as well as the underlying processes regulating elongation. The events that lead to promoter clearance and to the formation of early elongation complexes are intricately connected, and the third

1E-MAIL jones@salk.edu; FAX (619) 535-8194. new report in this issue by Cujec et al. supports and extends the recent findings of two other groups (Parada and Roeder 1996; Garcia-M artinez et al. 1997b) that the TFIIH kinase complex also plays an important role in Tat activation. Collectively, these studies highlight a critical and still largely undefined step in transcriptional control that constitutes a major regulatory checkpoint for the HIV-1 promoter as well as for many cellular genes.

\section{Transcriptional activation by Tat requires the CTD of RNAPII}

The HIV-1 and HIV-2 Tat proteins bind specifically to their respective transactivation response (TAR) RN A elements at the $5^{\prime}$ end of nascent viral transcripts to activate the RNAPII complex (for review, see Jones and Peterlin 1994). Tat contains a very strong transcriptional activation domain, composed of a Cys-rich region and a hydrophobic core motif (Fig. 1A), along with an argininerich RNA-binding motif (ARM) that specifies the binding of $T$ at to a base triple in the bulge region of the TAR RNA structure (Fig. 1B). Importantly, the Tat ARM is not an independent modular domain and is unable to target a heterologous protein to TAR RNA in vivo in the absence of the transactivation domain. Moreover, binding of purified Tat to TAR RNA does not require cisacting sequences in the loop of the TAR structure that have been shown to be critical for Tat activity in the cell. For these reasons, the interaction of Tat with a transcriptional coactivator is predicted to be required for highaffinity, loop-specific binding to TAR RN A. In contrast, the $T$ at transactivation domain can function independently of the ARM when tethered to the DNA- or RNAbinding domain of a heterologous protein, and on its own is a potent dominant negative inhibitor of the wild-type Tat protein, indicating that Tat can associate with its transcriptional coactivator independently of binding to TAR RNA.

Although Tat has been shown to have modest effects on the initiation of RN A synthesis, its primary role is to enhance the efficiency of elongation, and it has been noted that TAR-dependent transactivation by Tat is effectively blocked by the purine nucleoside analog DRB, an elongation inhibitor that inactivates protein kinases (Marciniak and Sharp 1991). Tat activation requires the CTD of RNAPII (Chun and Jeang 1996; Okamoto et al. 

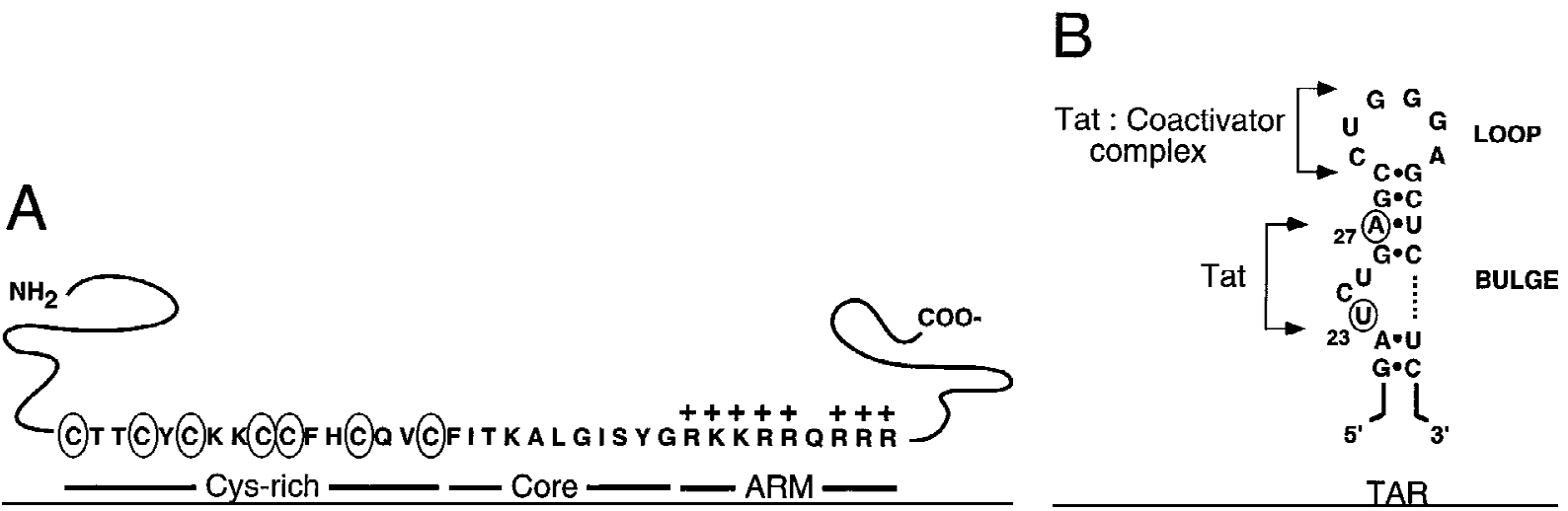

Figure 1. (A) D omain structure of the HIV T at proteins. (B) Sequence of the upper stem of the HIV-1 TAR RN A hairpin. Tat recognizes a base triple in the bulge formed by Hoogsteen interaction of U 23 with AU 27 in the upper stem (T ao et al. 1997). Sequences in the loop of TAR RNA are presumed to be recognized by a Tat:coactivator complex.

1996; Parada and Roeder 1996; Yang et al. 1996), which contains a tandemly reiterated heptapeptide sequence (YSPTSPS) that is differentially phosphorylated during the transcription cycle (for review, see Dahmus 1996). The CTD of RNAPII molecules present in preinitiation complexes or in early transcripti on complexes that have paused shortly downstream of the RNA start site is unphosphorylated (RN A Pol IIa), whereas active elongating polymerases contain exclusively hyperphosphorylated CTD structures (RNA Pol IIo). In general, hyperphosphorylation of the CTD is closely correlated with the generation of highly processive RNAPII molecules and may induce allosteric changes in the conformation of RNAPII that are conducive to elongation. CTD phosphorylation is al so thought to be critical for the rel ease of preinitiation complexes from the promoter and could disrupt contacts between the CTD and the mediator (Svejstrup et al . 1997) or other transcription factors, such as TATA-binding protein (TBP), that do not remain with the elongation complex (Zawel et al. 1995). Taken together, these findings suggest that the interaction between Tat and TAR affects a DRB-sensitive, CTD ki- nase-dependent step that is needed to form highly processive RNAPII elongation complexes.

\section{Interactions between Tat and the CTD kinase CAK (TFIIH)}

In support of this model, Tat has been shown to stimulate hyperphosphorylation of the CTD in a transcriptiondependent manner in vitro (Parada and Roeder 1996; Garcia-M artinez et al. 1997b). The cyclin-dependent kinase CDK7, present in the multisubunit TFIIH complex has been widely postulated to function as a major CTD kinase in vivo (for review, see Akoulitchev et al. 1995; Svejstrup et al. 1996), leading several groups to examine the possibility that Tat may target TFIIH directly (Fig. 2). TFIIH plays a critical role in transcription inititation and promoter clearance (Goodrich and Tjian 1994; Dvir et al. 1996) and is bound tightly to nonphosphorylated RN APII as an integral component of the RNAPII holoenzyme. The TFIIH complex dissociates from RNAPII 30-50 nucleotides after initiation and is not associated with elongating RN A polymerases (Zawel et al. 1995). Gen-
Figure 2. Properties of the CAK and TFIIH CTD kinase complexes. The CDK7 kinase is found in several different complexes in the cell, principally as a trimeric CAK complex containing the CDK7, Cyclin $\mathrm{H}$, and MAT 1 proteins. CDK7 associates with RN APII as part of a large multisubunit complex (TFIIH) containing DN A helicase and ATPase activies. Recent studies have implicated CAK/TFIIH as a coactivator for Tat (Parada and Roeder 1996; Cujec et al., this issue; Garcia-Martinez et al. 1997b).
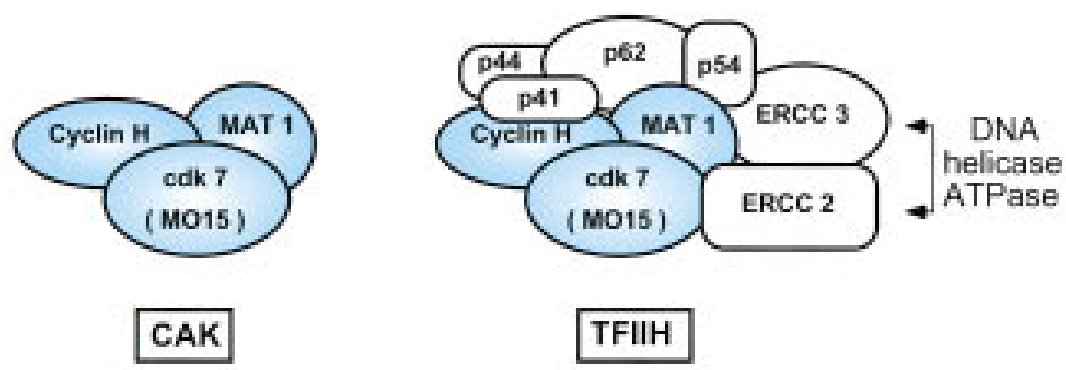

- TFIH prometes initiation and prometer clearance

- TFIIH / CAK stimulates hypophosphorylation of RNA polymerase II (pol lla)

- Tat enhances phosphorylation of the CTD by CAK in vitro

- TFliH and Tat activity is blocked by a cdk2 pseudosubstrate peptide inhibitor 
eral support for a role for TFIIH in the control of early transcription elongation derives from its sensitivity to the elongation inhibitor DRB and the observation that antibodies specific to TFIIH block elongation when injected into Xenopus oocytes (Yankulov et al. 1996; Dvir et al. 1997). A specific role for TFIIH in Tat transactivation has been suggested recently by immunodepletion experiments with antisera to various components of TFIIH that are necessary for Tat activity in vitro, and from the observation that $T$ at stimulates the phosphoryIation of the CTD by purified fractions of TFIIH (Parada and Roeder 1996; Garcia-M artinez et al. 1997b).

The current paper by Cujec et al. (this issue) extends these observations in two significant ways. First, wildtype but not mutant Tat proteins are shown to stimulate CTD phosphorylation by a fully recombinant CDK-activating kinase (CAK) complex in vitro. Second, TAT activity was blocked by a CDK2 substrate peptide containing a point mutation at Thr-160 that acts as a pseudosubstrate inhibitor of CDK7 and blocks CAK-mediated phosphorylation of the CTD in vitro. Importantly, the CDK 7 inhibitor dramatically reduced the formation of long transcripts that are dependent on Tat but had no effect on the short attenuated RNAs that form at the HIV-1 promoter independently of Tat. The specificity of the CDK7 pseudosubstrate inhibitor was apparent by its ability to block transcription in a promoter-selective manner. Similarly, overexpression of CDK7 preferentially stimulated long transcripts, and this effect was blocked with a kinase-deficient version of CDK7 (Cujec et al., this issue). Thus, the CDK7 complex associated with TFIIH appears to act at an early postinitiation step to assist in the transition to productive elongation, and may play an important role in Tat-induced transcription.

Despite this rather strong evidence that TFIIH is important for el ongation and Tat activity, a number of important details remain unresolved or contested. First, affinity-purified fractions of TFIIH (or recombinant CAK) predominantly induce a low extent of CTD phosphorylation rather than hyperphosphorylation of the CTD, even in the presence of Tat (Parada and Roeder 1996; Cujec et al., this issue). Therefore, other CTD kinases must be requi red to generate the characteristic pattern of CTD hyperphosphorylation observed in el ongation complexes, and these kinases could act independently or in a concerted manner with TFIIH. In this regard, it will be critical to establish whether the CDK7 pseudosubstrate inhibitor can also block the activity of other related kinases, especially P-TEFb (see below). A second, unresolved question concerns the mechanism whereby Tat engages TFIIH/CAK in the cell. Here, Cujec et al. (this issue) find that Tat binds directly and specifically to recombinant CDK 7 through its transactivation domain. In contrast, Parada and Roeder (1996) report that Tat interacts only indirectly with CDK7 through binding to the p62 subunit of TFIIH. Yet a different conclusion was reached by Garcia-Martinez et al. (1997b), who found that Tat binds and activates only a small fraction of CDK7 in extract that is associated with TFIIH (and not CAK) and further suggested that $T$ at interacts only with a large multisubunit complex containing components of TFIIH as well as novel cellular factors. In addition, other elongation factor and kinase complexes may contribute to Tat activation (Zhon and Sharp 1995, 1996). Thus, further studies are needed to identify the Tat-interacting regions of the TFIIH complex and to establish a direct role for CDK7 in the Tat-induced CTD hyperphosphorylation that is seen in crude extracts.

\section{Interactions between Tat and a new CTD kinase, TAK (P-TEFb)}

The concept that Tat may function by interacting with a cellular protein kinase was first suggested by Herrmann and Rice (1993, 1995), who demonstrated that a nuclear serine/threonine-directed kinase complex, called TAK (Tat-associated kinase), binds avidly and specifically to the Tat transactivation domain in vitro. Tat forms tight complexes with the multiprotein TAK complex in vivo (Yang et al. 1996), and this interaction was shown to correlate precisely with the ability of wild-type and mutant HIV-1, HIV-2, and equine infectious anemia virus (EIAV) Tat proteins to stimulate transcription in vivo. Affinity-purified TAK can hyperphosphorylate the RN APII CTD in vitro (Herrmann and Rice 1995; Y ang et al. 1996) and is unrelated to TFIIH or CDK8 (Herrmann et al. 1996). Pursuit of these intriguing observations has thus far been hampered by the lack of information regarding the molecular composition of the TAK complex, which is needed to establish a direct role for TAK in Tat activation in vivo.

The paper by Zhu et al. (this issue) now resolves this dilemma with the identification of PITALRE as the kinase responsible for TAK activity. Interestingly, this breakthrough originated from studies of P-TEFb (Kephart et al. 1992; Marshall and Price 1992), a positive-acting transcription elongation factor complex purified from Drosophila extracts (M arshall and Price 1995) that contains an associated kinase activity capable of hyperphosphorylating the RN APII CTD (Marshall et al. 1996). Microsequence analysis of the small subunit of the Drosophila P-TEFb revealed its near identity to the human PITALRE kinase (Zhu et al., this issue), and PITALRE was shown to be present in Tat-associated TAK complexes isol ated from HeLa cells and found to be indistinguishable from the 42-kD autophosphorylated TAK subunit originally identified by Herrmann and Rice (1993, 1995). Immunodepl etion of PIT ALRE from HeLa nucl ear extracts effectively blocked transcription elongation in vitro, which could be restored by Drosophila P-TEFb (Zhu, this issue). Purified Drosophila P-TEFb could not restore Tat activation to PITALRE-depleted extracts, however, indicating that Tat requires a PITALRE-associated factor that is not conserved in flies. These results clearly establish PITALRE as an essential component of a human P-TEFb (TAK) complex, which, like its Drosophila counterpart, plays an important role in enhancing the processivity of RNAPII. Moreover, these data strongly suggest that TAK (P-TEFb) is a functional target for the HIV-encoded Tat proteins. The steps that are pos- 
Figure 3. Properties of TAK (P-TEFb) CTD kinase (Kephardt et al. 1992; M arshall and Price 1992). Recent studies indicate that the Tat-associated kinase TAK (Herrmann and Rice 1993, 1995) is indistinguishable from the positive-acting early elongation factor P-TEFb (M ancebo et al.; Zhu et al., both this issue). PITALRE is the 42-kD catalytic subunit of TAK (P-TEFb) and contains a number of unidentified associated proteins, as indicated. These proteins do not necessarily form a single complex, and TAK activity in nuclear extracts principally migrates as a 110-kD complex (Yang et al. 1996), presumably consisting of PITALRE and its associated cyclin partner.
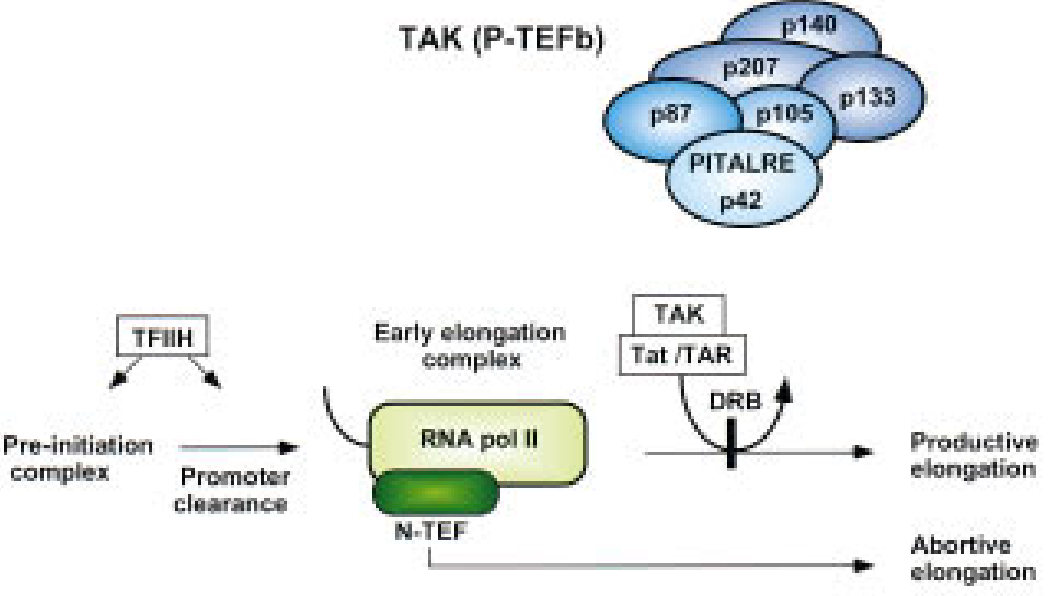

- TAK (P-TEFb) is required for the transition into productive elongation

- TAK (P-TEFb) stimulates hyperphosphorylation of RNA polymerase II

- TAK (P-TEFb) and Tat activation are blocked by DRB and novel TRB compounds

- TF IEH I CAK cannot substitute for TAK (P-TEFb) tulated to be regulated by Tat through P-TEFb (TAK) are indicated schematically in Figure 3.

Independent support for a role for P-TEFb and PITALRE in Tat activation comes from random drug screens described by M ancebo et al. (this issue). Remarkably, all of the inhibitors identified by this approach, including DRB, were found to be directed against kinases, and a strong correlation was observed between the ability of these inhibitors to block Tat activation and their corresponding effects on P-TEFb CTD kinase activity. Importantly, the inhibition profile of these compounds on CDK7/TFIIH activity did not correlate with their ability to block Tat activity. Although P-TEFb is a general transcription factor required for all elongation, low amounts of these inhibitors preferentially affected HIV-1 transcription over the expression of other promoters, indicating that the HIV-1 promoter in the presence of Tat is unusually susceptible to the action of these compounds. Overexpression of the PITALRE kinase augmented Tat activity in vivo, whereas a kinase-deficient PITALRE was found to block Tat activation, although this effect was only seen in one of three cell lines tested. Collectively, these papers demonstrate convincingly that PITALRE is the catalytic subunit of TAK (P-TEFb) and is likely to have a central role in Tat-mediated induction of transcription at the HIV-1 promoter.

PITALRE has been shown previously to be a widely expressed CDC2-related kinase that exhibits proline-directed serine/threonine activity and forms a multisubunit complex with several unknown proteins (Grana et al. 1994; Garriga et al. 1996), one of which will undoubtedly prove to be a cyclin partner for PITALRE. N either PITALRE nor Tat activity appears to be cell cycle-regulated (this is also true for TFIIH and CDK8), although basal HIV-1 promoter activity is up-regulated in HIV- infected cells arrested in $\mathrm{G}_{2}$ by the action of $\mathrm{Vpr}(\mathrm{M}$. Emerman, pers. comm.). Thus, the cyclin-dependent protein kinases implicated in transcription are not di rectly involved in cell-cycle control, although they may affect other pathways in the cell (Poon and Hunter 1995).

\section{Implications for the mechanism of Tat activation and TAR RNA recognition}

How should these disparate observations linking T at and the control of RNAPII elongation to two different CTD kinase complexes be regarded? M ost importantly, the involvement of Tat with TFIIH/CDK7 and/or TAK (PTEFb) kinases is necessarily mutually exclusive; rather, the studies outlined here raise the possibility that these two kinases may act in a concerted manner to promote hyperphosphorylation of the CTD in vivo (Fig. 4). Thus, $\mathrm{TFIIH}$, al one or compl exed with Tat, may stimulate lowlevel phosphorylation of the CTD at an early step in transcription prior to its release following promoter clearance, which could set the stage for the subsequent hyperphosphorylation of the CTD by P-TEFb. Previous studies have indicated that the P-TEFb kinase is more active on previously phosphorylated CTD substrates (M arshall and Price 1995; Marshall et al. 1996). The association of Tat with TFIIH could explain why Tat is associated with the RNAPII holoenzyme and preinitiation complexes prior to transcription (Cujec et al. 1997a; Garcia-Martinez et al. 1997a), and could underlie the modest effects of Tat on transcription initiation that have been observed in several systems. Alternatively, Tat may interact directly with the large subunit of RNAPII (Mavankal et al. 1996). TFIIH-associated Tat would exit the RNAPII complex prior to elongation (Zawel et al. 1995), which could explain why it is not 


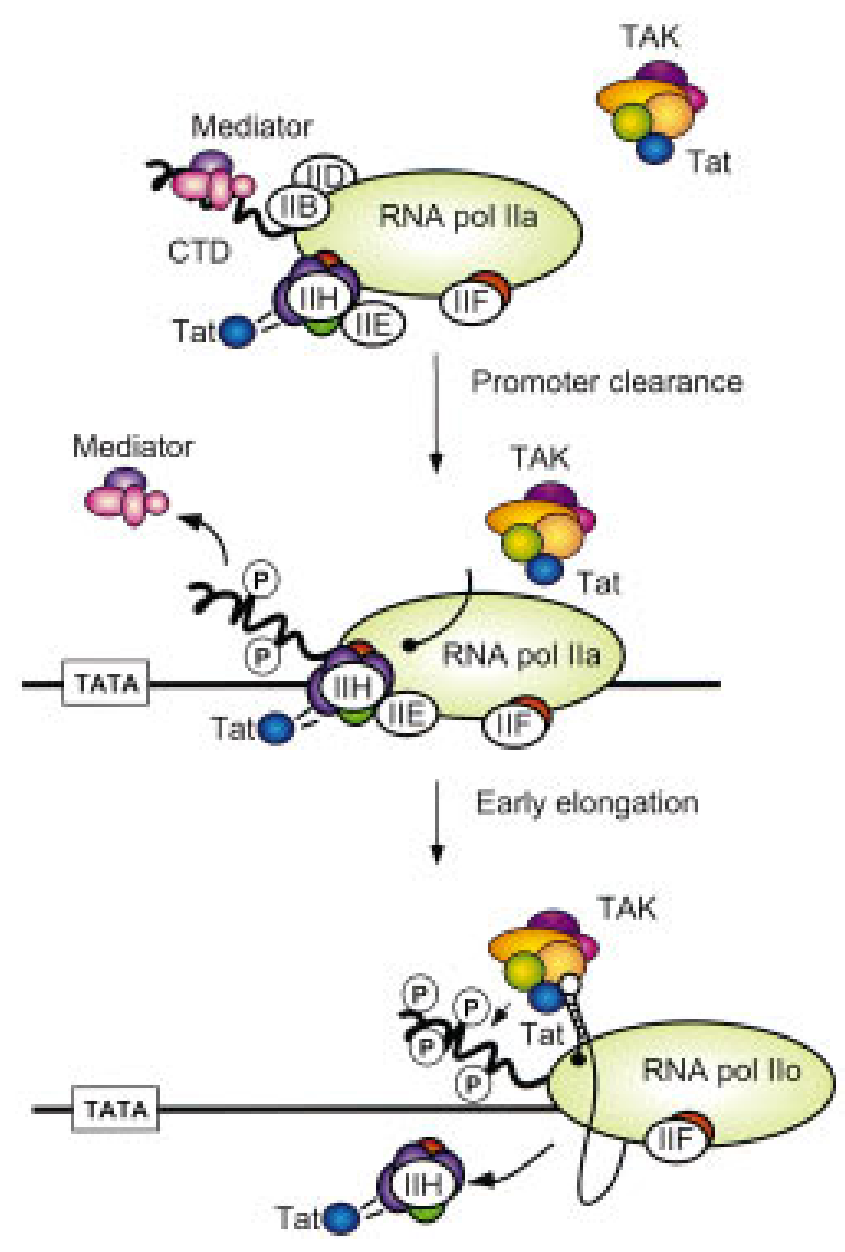

Figure 4. Hypothetical model of transcriptional activation by Tat.

detected on elongating RNAPII complexes in the absence of TAR (Keen et al. 1996).

In contrast with TFIIH, Tat forms a tight and specific complex with TAK in the absence of RNAPII or TAR. Because P-TEFb is a limiting transcription factor and is not associated with RNAPII initiation or elongation complexes (Marshall and Price 1995; Marshall et al. 1996), it is attractive to consider that Tat acts through binding to TAR to recruit P-TEFb to RNAPII and increase its local effective concentration at the promoter. The observation that Tat associates with elongating RNAPII complexes in the presence of TAR (Keen et al. 1996) indicates that TAK may also stabilize the association of Tat with TAR or the polymerase. A corollary of this model predicts that the TAK complex is not capable of interacting with TAR RN A in the absence of Tat. Tat is eventually released from TAR and associates with RN APII at later stages in el ongation (Keen et al. 1997). It is unlikely that Tat, TAR, or P-TEFb play any role in the maintenance of elongation at later stages in transcription, as this process is not sensitive to DRB, and because TAR need only be present transiently on the nascent transcript at early stages in transcription. It is interesting that Tat acts through RN A to regulate a step in el ongati on that apparently can otherwise be regulated by DN Abinding proteins (Blair et al. 1996; Blau et al. 1996). Two possible reasons for this are that regulation through RN A appears to be more effective (Blair et al. 1996) and is required to prevent a concomitant increase in the levels of short transcripts that contain TAR and would otherwise sequester Tat and its coactivator complex away from the promoter (Pendergrast and Hernandez 1997).

What is the role of CTD hyperphosphorylation in elongation? Importantly, studies on P-TEFb hint at the existence of a negative-acting transcription elongation factor(s) (N-TEFs) that restricts the formation of Iong transcripts without affecting short transcripts (Marshall and Price 1992). Similarly, Mancebo et al. (this issue) demonstrate that a negative activity present in nuclear extracts must be added to a purified reconstituted transcription system to reconstitute appropriate regulation of T at through P-TEFb. Given that P-TEFb is now shown to be a CDC2-related kinase, this N-TEF activity could in principle be a CDK inhibitor or a CTD phosphatase. However, previous studies on N-TEF indicate that this activity does not require the CTD (Marshall and Price 1992); therefore, the negative factors could act on any part of the transcription complex. One component of $\mathrm{N}$ TEF, factor 2, is an ATP-dependent RN APII termination factor associated with early elongation complexes (Xie and Price 1996), which functions through a direct interaction with DNA (Z. Xie and D.H. Price, pers. comm.). Another transcriptional activity that could compete with Tat is the DNA-binding activity associated with the IST (inducer of short transcripts), which stimulates attenuatéd transcri pts without affecting the synthesis of long transcripts (Pessler et al. 1997). Theidentification of these various negative effectors of elongation will be critical for a complete understanding of the role of PTEFb (TAK) and Tat in transcriptional regulation.

\section{Other implications and perspectives}

The identification of new CTD kinase cofactors that interact with $\mathrm{T}$ at has many important implications for $\mathrm{T}$ at function in infected cells. First, if TAK (P-TEFb) is the coactivator necessary for $T$ at to function through TAR, the Tat:TAK complex should bind TAR RNA with an appropriate loop sequence specificity that is not observed with Tat al one (for revi ew, see Jones and Peterlin 1994). Genetic studies further predict that a protein(s) in the TAK complex may play a role in the observed species-specific restriction of Tat activity. Tat cannot function effectively through TAR in nonpermissive rodent cells (Hart et al. 1993; Alonso et al. 1994), and the identification of human factors necessary for TAR recogniti on would be an important step toward the development of transgenic mice that can be infected with HIV-1 to model AIDS disease progression and its consequences on the immune system. Second, the identification of the protein in the TAK (P-TEFb) complex that interacts with 
Tat could yield new inhibitors of Tat that specifically block its association with TAK. Compounds such as these and others that block the ability of Tat to interact with TAR RN A (Hamy et al. 1997) may have fewer undesired effects on the expression of host cell genes than compounds such as DRB that target the active site of the PITALRE kinase. Third, it will be important to learn whether the activity of TAK or TFIIH is stimulated by T-cell activation, which dramati cally up-regulates HIV-1 transcription, and, if so, which cellular signaling pathways (Hunter 1997) regulate these CTD kinases. Fourth, the interaction of Tat with TAK and TFIIH may play a role in the demonstrated ability of Tat to regulate cytokine gene expression and immune cell hyperactivation (Ott et al. 1997), as well as its ability to induce apoptosis in infected and bystander cells (Li et al. 1995).

Beyond these specific implications for Tat activity, it will be of great interest to learn whether the P-TEFb complex is also targeted by cellular transcription factors that control the regulation of the growing class of cellular genes (including heat shock, c-myc, c-fos, and other oncogenes) that have been shown to be regulated by attenutation. A recent study suggests that E1A and VP16 may enhance elongation through binding to CDK8 rather than TAK (Gold et al. 1996), suggesting that diverse activators may use different means to enhance CTD hyperphosphorylation. Given that PITALRE has an associated cyclin, it should by convention be renamed CDK9, and its partner, cyclin K. The coming year should provide many more exciting insights into the varied roles of the three CTD kinases, CDK7, CDK8, and CDK9.

\section{Acknowledgments}

I thank Tony Hunter and James T. Kadonaga for their insightful comments on this review and apologize to those whose work could not be cited directly because of space limitations.

\section{References}

Akoulitchev, S., T. M akela, R. Weinberg, and D. Reinberg. 1995. Requirement for TFIIH kinase activity in transcription by RN A polymerase II. Nature 377: 557-560.

Alonso, A., T. Cujec, and B. Peterlin. 1994. Effects of human chromosome 12 on interactions between Tat and TAR of human immunodeficiency virus type 1. J. Virol. 66: 65056513.

Bentley, D. 1995. Regulation of transcriptional elongation by RN A polymerase II. Curr. Opin. Genet. Dev. 5: 210-216.

Blair, W., R. Fridell, and B. Cullen. 1996. Synergistic enhancement of both initiation and elongation by acidic transcription activation domains. EMBO J. 15: 1658-1665.

Blau, J., H. Xiao, S. M cCracken, P. O'Hare, J. Greenblatt, and D. Bentley. 1996. Three functional classes of transcriptional activation domains. Mol. Cell. Biol. 16: 2044-2055.

Chun, R. and K. Jeang. 1996. Requirements for RN A polymerase II carboxyl-terminal domain for activated transcription of human retroviruses human $\mathrm{T}$-cell lymphotrophic virus I and HIV-1. J. Biol. Chem. 271: 27888-27894.

Cujec, T., H. Cho, E. Maldonado, J. Meyer, D. Reinberg, and B. Peterlin. 1997a. The human immunodeficiency virus trans- activator Tat interacts with the RNA polymerase II holoenzyme. Mol. Cell. Biol. 17: 1817-1823.

Cujec, T., H. Okamoto, K. Fujinaga, J. M eyer, H. Chamberlin, D. M organ, and B. Peterlin. 1997b. The HIV trans-activator Tat binds to the CDK-activating kinase (CAK) and activates the phosphorylation of the C-terminal domain of RNA polymerase II. Genes \& Dev. (this issue).

Dahmus, M. 1996. Reversible phosphorylation of the C-terminal domain of RN A polymerase II. J. Biol. Chem. 271: 1900919012.

Dvir, A., K. Garrett, C. Chalut, J.-M. Egly, J. Conaway, and R. Conaway. 1996. A role for ATP and TFIIH in activation of the RN A polymerase II preinitiation complex prior to transcription initiation. J. Biol. Chem. 271: 7245-7248.

Dvir, A., R. Conaway, and J. Conaway. 1997. A role for TFIIH in controlling the activity of early RNA polymerase II elongation complexes. Proc. Natl. Acad. Sci. 94: 9006-9010.

Garcia-M artinez, L., D. Ivanov, and R. Gaynor. 1997a. Association of Tat with purified HIV-1 and HIV-2 transcription preinitiation complexes. J. Biol. Chem. 272: 6951-6958.

Garcia-M artinez, L., G. M avankal, J. N eveu, W. Lane, D. Ivanov, and R. Gaynor. 1997b. Purification of a Tat-associated kinase reveals a TFIIH complex that modulates HIV-1 transcription. EMBO J. 16: 2836-2850.

Garriga, J., X. Mayol, and X. Grana. 1996. The CDC2-related kinase PITALRE is the catalytic subunit of active multimeric protein complexes. Biochem. J. 319: 293-298.

Gold, M., J.-P. Tassan, E. Nigg, A. Rice, and C. Herrmann. 1996. Viral transactivators E1A and VP16 interact with a large complex that is associated with CTD kinase activity and contains CDK8. Nucleic Acids Res. 24: 3771-3777.

Goodrich, J. and R. Tjian. 1994. Transcription factors IIE and IIH and ATP hydrolysis direct promoter clearance by RN A polymerase II. Cell 77: 145-156.

Grana, X., A. De Luca, N. Sang, Y. Fu, P. Claudio, J. Rosenblatt, D. Morgan, and A. Giordano. 1994. PITALRE, a nuclear CDC2-related protein kinase that phosphorylates the retinoblastoma protein in vitro. Proc. Natl. Acad. Sci. 91: 38343838.

Greenblatt, J., J.R. N odwell, and S.W. M ason. 1993. Transcriptional antitermination. Nature 364: 401-416.

Hamy, F., E. Felder, G. Heizmann, J. Lazdins, F. Aboul-ela, G. Varani, J. Karn, and T. Klimkai. 1997. An inhibitor of the Tat/TAR RN A interaction that effectively suppresses HIV-1 replication. Proc. Natl. Acad. Sci. 94: 3548-3553.

Hart, C., J. Gal phin, M. Westhafer, and G. Schochetman. 1993. TAR loop-dependent human immunodeficiency virus trans activation requires factors encoded on human chromosome 12. J. Virol. 67: 5020-5024.

Herrmann, C. and A. Rice. 1993. Specific interaction of the human immunodeficiency virus Tat proteins with a cellular protein kinase. Virology 197: 601-608.

- - - 1995. Lentivirus Tat proteins specifically associate with a cellular protein kinase, TAK, that hyperphosphorylates the carboxyl-terminal domain of the large subunit of RNA polymerase II: candidate for a Tat cofactor. J. Virol. 69: 16121620.

Herrmann, C., M. Gold, and A. Rice. 1996. Viral transactivators specifical ly target distinct cellular protein kinases that phosphorylate the RNA polymerase II C-terminal domain. Nucleic Acids Res. 24: 501-508.

Hunter, T. 1997. Oncoprotein networks. Cell 88: 333-346.

Jones, K. and B. Peterlin. 1994. Control of RNA initiation and el ongation at the HIV-1 promoter. Annu. Rev. Biochem. 63: 717-743.

Keen, N., M. Gait, and J. Karn. 1996. Human immunodeficiency 
virus type- 1 Tat is an integral component of the activated transcription-el ongation complex. Proc. Natl. Acad. Sci. 93: 2505-2510.

Keen, N., M. Churcher, and J. Karn. 1997. Transfer of Tat and release of TAR RNA during the activation of the human immunodeficiency virus type-1 transcription elongation complex. EMBO J. 16: 5260-5272.

Kephart, D., N. M arshall, and D. Price. 1992. Stability of Drosophila RNA polymerase II elongation complexes in vitro. Mol. Cell. Biol. 12: 2067-2077.

Li, C., D. Friedman, C. Wang, V. Metelev, and A. Pardee. 1995. Induction of apoptosis in uninfected lymphocytes by HIV-1 Tat protein. Science 268: 429-431.

Mancebo, H., G. Lee, J. Flygare, J. Tomassini, P. Luu, Y. Zhu, C. Blau, D. Hazuda, D. Price, and O. Flores. 1997. P-TEFb kinase is required for HIV Tat transcriptional activation in vivo and in vitro. Genes \& Dev. (this issue).

Marciniak, R. and P. Sharp. 1991. HIV-1 Tat promotes formation of more-processive elongation complexes. EMBO J. 10: 4189-4196.

Marshall, N. and D. Price. 1992. Control of formation of two distinct classes of RN A polymerase II el ongation complexes. Mol. Cell. Biol. 12: 2078-2090.

- - - 1995. Purification of P-TEFb, a transcription factor required for the transition into productive elongation. J. Biol. Chem. 270: 12335-12338.

M arshall, N ., J. Peng, Z. Xie, and D. Price. 1996. Control of RN A polymerase II elongation potential by a novel carboxyl-terminal domain kinase. J. Biol. Chem. 271: 27176-27183.

Mavankal, G., S. Ignatius Ou, H. Oliver, D. Sigman, and R. Gaynor. 1996. Human immunodeficiency virus type 1 and 2 Tat proteins specifically interact with RNA polymerase II. Proc. Natl. Acad. Sci. 93: 2089-2094.

Okamoto, H., C. Sheline, J. Corden, K. Jones, and B. Peterlin. 1996. Trans-activation by human immunodeficiency virus Tat protein requires the C-terminal domain of RNA polymerase II. Proc. Natl. Acad. Sci. 93: 11575-11579.

Ott, M., S. Emiliani, C. Van Lint, G. Herbein, J. Lovett, N. Chirmule, T. McCloskey, S. Pahwa, and E. Verdin. 1997. Immune hyperactivation of HIV-1-infected T cells mediated by Tat and the CD28 pathway. Science 275: 1481-1485.

Parada, C. and R. Roeder. 1996. Enhanced processivity of RN A polymerase II triggered by Tat-induced phosphorylation of its carboxyl-terminal domain. Nature 384: 375-378.

Pendergrast, P. and N. Hernandez. 1997. RN A-targeted activators, but not DN A-targeted activators, repress the synthesis of short transcripts at human immunodeficiency virus type 1 long terminal repeat. J. Virol. 71: 910-917.

Pessler, F., P. Pendergrast, and N. Hernandez. 1997. Purification and characterization of FBI-1, a cellular factor that binds to the human immunodeficiency virus type 1 inducer of short transcripts. Mol. Cell. Biol. 17: 3786-3798.

Poon, R. and T. Hunter. 1995. Innocent bystanders or chosen collaborators? Curr. Biol. 5: 1243-1247.

Svejstrup, J., P. Vichi, and J.-M . Egly. 1996. The multiple roles of transcription/repair factor TFIIH. Trends Biochem. Sci. 21: 346-350.

Svejstrup, J., Y. Li, J. Fellows, A. Gnatt, S. Bjorklund, and R. Kornberg. 1997. Evidence for a mediator cycle at the initiation of transcription. Proc. Natl. Acad. Sci. 94: 6075-6078.

Tao, J., L. Chen, and A. Frankel. 1997. Dissection of the proposed base triple in human immunodeficiency virus TAR RNA indicates the importance of the Hoogsteen interaction. Biochemistry 36: 3491-3495.

Xie, Z. and D.H. Price. 1996. Purification of an RN A polymerase II transcript release factor from Drosophila J. Biol. Chem.
271: 11043-11046.

Yang, X., C. Herrmann, and A. Rice. 1996. The human immunodeficiency virus Tat proteins specifically associate with TAK in vivo and require the carboxyl-terminal domain of RNA polymerase II for function. J. Virol. 70: 4576-4584.

Yankulov, K., M. Pandes, S. McCracken, D. Bouchard, and D. Bentley. 1996. TFIIH functions in regulating transcriptional elongation by RNA polymerase II in Xenopus oocytes. Mol. Cell. Biol. 16: 3291-3299.

Zawel, L., K.P. Kumar, and D. Reinberg. 1995. Recycling of the general transcription factors during RN A polymearse II transcription. Genes \& Dev. 9: 1479-1490.

Zhou, Q. and P.A. Sharp. 1995. N ovel mechanism and factor for regulation by HIV-1 Tat. EMBO J. 14: 321-328.

-_- 1996. Tat-SF1: Cofactor for stimulation of transcriptional el ongation by HIV-1 Tat. Science 274: 605-610.

Zhu, Y., T. Pe'ery, J. Peng, Y. Ramanathan, N. Marshall, T. Marshall, B. Amendt, M. M athews, and D. Price. 1997. Transcription el ongation factor $\mathrm{P}$-TEFb is required for HIV-1 Tat transactivation in vitro. Genes \& Dev. (this issue). 


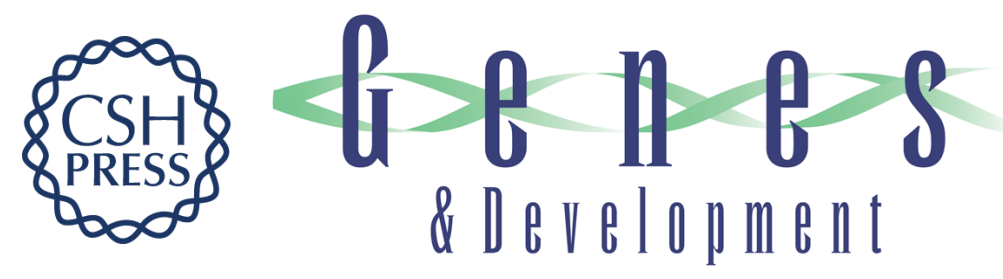

\section{Taking a new TAK on Tat transactivation}

Katherine A. Jones

Genes Dev. 1997, 11:

Access the most recent version at doi:10.1101/gad.11.20.2593

References This article cites 47 articles, 30 of which can be accessed free at: http://genesdev.cshlp.org/content/11/20/2593.full.html\#ref-list-1

License

Email Alerting Receive free email alerts when new articles cite this article - sign up in the box at the top Service right corner of the article or click here.

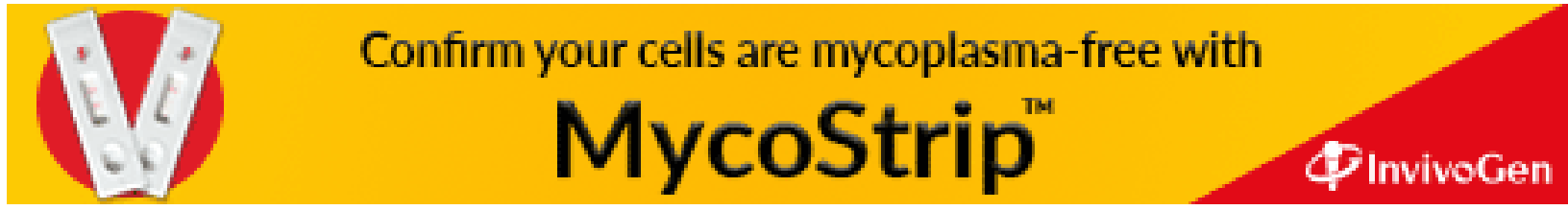

